\title{
Advances in ALK Targeted Therapy for Neuroblastoma
}

\section{Libo Zhang ${ }^{1,2 *}$ and Sylvain Baruchel ${ }^{3,4}$}

${ }^{1}$ Department of Molecular Medicine, The Hospital for Sick Children, Toronto, Canada

${ }^{2}$ Department of Anesthesia, The Hospital for Sick Children, Toronto, Canada

${ }^{3}$ Deptartment of Pediatrics, The Hospital for Sick Children, Toronto, Canada

${ }^{4}$ Institute of Medical Sciences, University of Toronto, Toronto, Canada

\begin{abstract}
Significant advances have been made to understand the association between ALK genetic aberrations and disease prognosis in neuroblastoma. ALK targeted therapies are evolving quickly and several randomized controlled trials of ALK inhibitors are underway or nearing completion in adult cancers. Ongoing research will bring new challenges and newer technologies to fully define the pathogenic and prognostic alterations, to stratify the risk of recurrence or progression, and to develop optimal monitoring and treatment strategies in this malignancy.
\end{abstract}

Keywords: ALK; Neuroblastoma; Drug treatment

\section{Introduction}

Neuroblastoma is the most common extracranial solid tumor originating from the sympathoadrenal lineage of the neural crest. It has extensive pathologic and molecular heterogeneity which decides the significant clinical diversity from spontaneous regression to highlyaggressive and drug resistant metastatic disease. Although multimodal chemotherapy/radiotherapies/immunotherapy significantly improves patient survival during the last few decades, some patients will continue to relapse and die of this malignancy because of de novo or acquired drug resistance, especially for high-risk neuroblastoma patients [1,2].

Over the last decades, concerted efforts have been made to identify oncogenic alterations in subsets of neuroblastoma, such as molecular alterations of MYCN, anaplastic lymphoma kinase (ALK), paired-like homeobox 2B (PHOX2B), etc. [3-6]. Next-generation sequencing-based genomic profiling identified the most frequent alterations including MYCN (26.5\%), ALK (17.8\%), ATRX (6.5\%), CDKN2A (4.8\%) and RPTOR (4.8\%) in 230 neuroblastoma patient samples [7]. Both ALK and MYCN genes are located in chromosome $2 \mathrm{p}$, a chromosomal alteration identified as a statistically significant prognostic factor [8]. It has been shown that ALK and MYCN drive tumor malignancy cooperatively. Activation of ALK increases the expression of MYCN by enhancing the activity of the MYCN promoter and stabilizing MYCN protein likely via activation of AKT and ERK pathways [9-11]. In vivo, compared to ALKF1174L and MYCN alone, co expression of these two oncogenes leads to the development of neuroblastoma tumors with earlier onset, higher penetrance and enhanced lethality $[10,12,13]$. In our recent study, neuroblastoma cells harboring both ALKF1174L mutation and MYCN amplification showed less responsive to an ALK inhibitor, crizotinib, comparing to other variants [14]. Understanding those tumor-specific, oncogenic driver mutations would provide further insight into the biology of this disease and transformed our treatment strategy into the era of precision medicine.

\section{ALK Variants in Neuroblastoma}

Among all those identified oncogenic mutations, ALK is one of the well-studied druggable molecular targets. ALK was first discovered in 1994 as a fusion protein with nucleophosmin (NPM) in a subset of anaplastic large-cell lymphomas (ALCLs) as a result of $\mathrm{t}(2 ; 5)(\mathrm{p} 23 ; \mathrm{q} 35)$ chromosomal translocation [15]. In 2007, ALK gene rearrangements were reported in a subset of patients with non-small cell lung cancer (NSCLC) [16]. Since then, ALK mutations have been regarded as oncogenic mutations. More mutations of ALK gene have been reported in different cancer types, including NSCLC, inflammatory myofibroblastic tumors, colon cancer, renal cell carcinoma, breast carcinoma and esophageal cancer [17]. In 2008, several groups discovered ALK mutations, including germline missense mutations and somatically acquired mutations, in high-risk neuroblastoma patients [18-20]. They also found that mutated kinases were autophosphorylated and displayed increased kinase activity compared with the wild-type ALK.

So far, more than 35 ALK variants have been detected in neuroblastoma, predominantly point mutations [21], with fewer cases of truncated extracellular domain [22,23] and BEND5-ALK fusion protein [7]. ALK mutations are found in almost all cases of familial neuroblastoma $(<2 \%$ of all neuroblastoma) [24]. There are three most common pathogenic variants ALKR1275Q, ALKG1128A and ALKF1174L identified in familial neuroblastoma patients, among which ALKR1275Q accounts for 45\% of ALK germline mutation $[4,18]$. ALK mutations have also been reported in about $6-10 \%$ of sporadic neuroblastoma cases [25]. 12 different ALK active mutations have been reported in sporadic neuroblastoma, including two most common ALK variants, ALKF1174L and ALKR1275Q [26]. Most of these pathogenic variants are found within the tyrosine kinase domain of ALK and cause constitutive autophosphorylation and activation of the ALK protein and downstream cellular pathways, including the MAPK and RASrelated protein 1 signal pathways $[9,27]$. The PI3K (phosphatidylinositol 3-kinase)/Akt [10] and the JAK/STAT (Janus activated kinase/signal transducer and activator of transcription) pathways [28].

\section{ALK Targeted Agents in Neuroblastoma}

In 2011, crizotinib (PF-02341066, Xalkori) became the first FDAapproved ALK inhibitor for ALK-positive NSCLC based on strong

*Corresponding author: Sylvain Baruchel, Innovative Therapeutic Trials Office, Dept. of Pediatrics and Research Institute, The Hospital for Sick Children, 555 University Avenue, Toronto, Ontario, Canada M5G 1X8, Tel: 416-813-7795; Fax 416-813-5327; E-mail: sylvain.baruchel@sickkids.ca

Received July 27, 2017; Accepted August 04, 2017; Published August 21, 2017

Citation: Zhang L, Baruchel S (2017) Advances in ALK Targeted Therapy for Neuroblastoma. J Oncol Transl Res 3: 114. doi: 10.4172/2476-2261.1000114

Copyright: @ 2017 Zhang L, et al. This is an open-access article distributed under the terms of the Creative Commons Attribution License, which permits unrestricted use, distribution, and reproduction in any medium, provided the original author and source are credited. 
clinical response data [29]. It is an oral small-molecule tyrosine kinase inhibitor, originally developed as a c-MET inhibitor and later found an inhibitor for ALK phosphorylation [30,31]. In vitro studies demonstrated that crizotinib is potent in neuroblastoma cell lines with ALK amplification or the R1275Q mutation, one of the most common ALK variants in neuroblastoma [32]. Whereas cells bearing ALKF1174L mutation are relatively crizotinib-resistant [18,19,33,34]. In vivo, crizotinib treatment causes complete and sustained regression of xenografts with ALKR1275Q mutation, but it has limited effects on the growth of ALKF1174L-positive tumors [21]. Crizotinib has been tested in Phase I clinical trial for the treatment of pediatric solid tumors and it is currently being tested in a subset of neuroblastoma bearing ALK mutations and rearrangements [35].

Same as other tyrosine kinase inhibitors, crizotinib invariably loses its potency and drug resistance emerges after initial successful crizotinib treatment. Crizotinib resistance may develop in multiple tumor types as a consequence of secondary mutations in the ALK tyrosine kinase domain, the activation of other bypass pathways, or amplification of the ALK locus [36,37].

Epithelial-mesenchymal transition (EMT) also contributes to resistance to crizotinib in lung cancer cells [38], especially for secondgeneration ALK inhibitors [39]. In neuroblastoma, ALKF1174L mutation serves as a common cause of resistance to crizotinib, due to the increased ATP-binding affinity of this mutant [40]. ALK F1174L mutation also causes resistance to other ALK inhibitors. Acquired resistance to TAE684 and LDK378 was observed in ALKF1174 mutant human neuroblastoma cells, which is associated with AXL activation and induction of EMT [41]. Some other mechanisms could also regulate the sensitivity of ALK inhibitors in neuroblastoma. A recent study with genome-wide microRNA profiling identified that microRNAs, miR-424-5p and miR503-5p, were involved in regulating ALK expression, which may serve as potential therapeutic tools in ALK dependent neuroblastoma [42]. In some cases, crizotinib resistance may also arise from pre-existing minority cell populations with drug resistance due to intratumor heterogeneity in neuroblastoma tumors. Yan et al. [43] identified a subpopulation of neuroblastoma cells that are insensitive to the ALK kinase inhibitor. These cells have Schwann cell-like features, possess unique signaling profiles, but express the undetectable level of ALK. Treatment of SK-N-SH with an ALK kinase inhibitor TAE684 results in the outgrowth of the S-type cells. These TAE684-resistant S-type cells are also believed to protect N-type cells against the apoptotic effect of an ALK kinase inhibitor through upregulating prosurvival signaling [43].

In order to settle crizotinib resistance, several other ALK inhibitors, including ceritinib, brigatinib (AP26113), alectinib, lorlatinib (PF6463922), ensartinib (X-396), entrectinib (RXDX-101), and belizatinib (TSR 011), have been developed in clinical use for adult patients $[44,45]$.

Among those ALK inhibitors, only ceritinib and lorlatinib have been tested in clinical trials for pediatric patients. Ceritinib was approved by FDA in 2014 for the treatment of patients with ALKpositive lung cancer who relapse after first-line therapy [46]. It overcomes some crizotinib- resistant mutations, but ALK tumors harboring the ALKF1174L mutation still exhibit resistance to ceritinib [47,48]. Lorlatinib, as a selective next-generation ROS1/ALK inhibitor, has high potency across ALK variants, ALKR1275Q, ALKF1174L and ALKF1245C mutations. It induces complete tumor regression in both crizotinib-resistant and crizotinib-sensitive neuroblastoma xenograft models, as well as in patient-derived xenografts [45]. A new ALK/ IGF1R inhibitor AZD3463 was designed by AstraZeneca to overcome the acquired resistance to crizotinib.
This new drug suppressed cell proliferation of neuroblastoma cell lines with wild type ALK as well as ALK activating mutations (ALKF1174L and ALKD1091N) by blocking the ALK-mediated PI3K/AKT/mTOR pathway. In addition, AZD3463 also exhibited significant therapeutic effects on the growth of the NB tumors bearing an ALKF1174L mutation in orthotopic xenograft mouse models [47]. Most recently, A novel ALK inhibitor alectinib (5-chloro-2,4diaminophenylpyrimidine) has been tested in neuroblastoma preclinical models and showed substantial inhibitory effects against tumors with ALK mutations, including ALKL1152R, ALKF1174L and ALKD1091N $[49,50]$. Although the new generation ALK inhibitors overcome crizotinib-resistant ALK mutations, patients almost invariably relapse. Genotype assessment of repeat biopsies from ALK-positive patients progressing on various ALK inhibitors revealed that only a minority of ALK-positive patients ( 20\%) developed ALK resistance mutations on crizotinib, while ALK resistance mutations were present in over one-half of patients progressing on second-generation ALK inhibitors. Also, the spectrum of ALK mutations was different following secondgeneration ALK inhibitors compared to crizotinib [39]. New and alternative approaches are required to tackling drug resistance against ALK inhibitors.

\section{Combined Therapy with ALK Inhibitors}

One of the mechanisms by which cancer cells evade kinase inhibitor-induced apoptosis is to switch to alternative signaling pathways. Combinatorial approaches to inhibit multiple kinases could be a therapeutic possibility to reverse drug resistance acquired from activation of bypass pathways. To overcome the drug resistance secondary to ALK inhibitors, Krytska et al. [51] combined crizotinib with the chemotherapeutic agents and showed increased cytotoxic effects comparing to crizotinib or chemotherapy alone in vitro. Combined therapy also restored sensitivity in preclinical models harboring ALK aberrations (both mutation and amplification). Hypoxia regulates tumor cell proliferation, migration and invasiveness through the expression of a group of transcription factors called hypoxiainducible factors (HIFs) [52,53]. It has been demonstrated that ALK specifically regulates HIF-1 $\alpha$ expression under hypoxia conditions in both ALCL and NSCLC [54]. Topotecan, especially daily metronomic topotecan, induces oxidative stress and down-regulates HIF-1 alpha expression in cancer cells [55-57]. In our recent study, single-agent crizotinib showed limited anti-tumor activity in ALKF1174L-mutated neuroblastoma xenograft models, however when combined with topotecan, significantly delayed tumor development was achieved. This anti-tumor activity was achieved through targeting of this hypoxia related pathway. In addition, relapsed tumors remained responsive to combined therapy [14]. Synergistic antitumor activities have also been observed when combining ALK inhibitor ceritinib with a dual inhibitor of cyclin-dependent kinase CDK4 and CDK6 [58] or with an MDM2 inhibitor NVP-CGM097 which targets p53 for proteasome-mediated degradation [59].

\section{Future Directions - Precision Medicine in Neuroblas- toma}

Targeted agents for different ALK variants improved clinical outcomes over the last few years, with newer agents rapidly entering clinical practice. However, ALK genetic alterations are not routinely evaluated in neuroblastoma standard clinical practice. Newer technologies are being introduced to fully define the pathogenic and prognostic impact of ALK alterations in neuroblastoma, as well as the emerging importance for therapeutic purposes. Lodrini et al. [60] 
established droplet digital PCR (ddPCR) protocols for MYCN and ALK copy number status in cell-free neuroblastoma-derived DNA isolated from plasma of neuroblastoma patients. They accurately discriminated between MYCN and ALK amplification, gain and normal diploid status in conditioned culture medium, mouse plasma from xenograft models, as well as NB patient plasma [60]. Tucker et al. [61] used ALK immunoassays to detect ALK and phosphorylated ALK and revealed a quantitative difference in on-target pharmacodynamic changes between a first- and second-generation ALK inhibitor, crizotinib and ceritinib, which will potentially become a valuable tool in preclinical and clinical evaluation in defining active doses and optimum treatment schedules [61].

Precision medicine has become a new model of health care aimed at tailoring therapies to an individual's genetic profile. As technologies and therapies improve, retrospective and prospective studies of cohorts of neuroblastoma patients are being required to incorporate genetic analyses into clinical practice beyond diagnostic purposes. A retrospective study by Padovan-Merhar et al. [62] defined the frequency of genomic alterations by gene panel sequencing in neuroblastoma patients, both at diagnosis and after chemotherapy and showed a higher frequency of ALK mutations in relapsed disease than at diagnosis. Suspected driver ALK variants were present in 3/43 (7.0\%) of samples at diagnosis, $7 / 41(17 \%)$ post-treatment samples, and in 11/54 (20\%) of samples at relapse. The same trend was observed in some other tumor-related genes, particularly for the alterations in the RAS/MAPK pathway, in relapsed high-risk neuroblastoma [62].

\section{Conclusion}

A better understanding of the switch to alternative signaling pathways will lead to strategies to bypass drug resistance. This proof of principle may also apply to other genetic alterations such as MYCN, NRAS, NTRK2/TrkB, etc. Given the growing number of genetic alterations detected, small sequencing panels that focus on a limited number of genes may not be sufficient, especially in highly heterogeneous neuroblastoma tumors. Further improvements in next-generation sequencing technologies are expected to allow the evaluation of genetic variants across the entire genome, which is also a more straightforward strategy for mapping mutations.

The molecular profile of each individual patient at different stages of treatment will inform physicians the detailed genetic condition and enable personalized targeted therapeutic interventions in specific subsets of neuroblastoma patients.

\section{Disclosure of Potential Conflicts of Interest}

Dr. Sylvain Baruchel participates in consulting activities for NeoMed Inc. (Montreal, Quebec).

\section{References}

1. Matthay KK, Villablanca JG, Seeger RC, Stram DO, Harris RE, et al (1999). Treatment of high-risk neuroblastoma with intensive chemotherapy, radiotherapy, autologous bone marrow transplantation and 13-cis-retinoic acid. Children's Cancer Group. N Engl J Med 341: 1165-1173.

2. Pearson AD, Pinkerton CR, Lewis IJ, Imeson J, Ellershaw C, et al. (2008) High-dose rapid and standard induction chemotherapy for patients aged over 1 year with stage 4 neuroblastoma: A randomised trial. Lancet Oncol 9: 247-256.

3. Trochet $D$, Bourdeaut $F$, Janoueix-Lerosey I, Deville A, de Pontual L, et al. (2004) Germline mutations of the paired-like homeobox 2B (PHOX2B) gene in neuroblastoma. Am J Hum Genet 74: 761-764.

4. Janoueix-Lerosey I, Lequin D, Brugieres L, Ribeiro A, de Pontual L, et al. (2008). Somatic and germline activating mutations of the ALK kinase receptor in neuroblastoma. Nature 455: 967-970.
5. Schleiermacher G, Janoueix-Lerosey I, Delattre O (2014) Recent insights into the biology of neuroblastoma. Int J Cancer 135: 2249-2261.

6. Campbell K, Gastier-Foster JM, Mann M, et al. (2017)Association of MYCN copy number with clinical features, tumor biology, and outcomes in neuroblastoma: A report from the Children's Oncology Group.

7. Chmielecki J, Bailey M, He J, Elvin J, Vergilio JA, et al. (2017) Genomic profiling of a large set of diverse pediatric cancers identifies known and novel mutations across tumor spectra. Cancer Res 77: 509-519.

8. Janoueix-Lerosey I, Schleiermacher G, Michels E, Mosseri V, Ribeiro A, et al. (2009). Overall genomic pattern is a predictor of outcome in neuroblastoma. $J$ Clin Oncol 27: 1026-1033.

9. Schönherr C, Ruuth K, Kamaraj S, Wang CL, Yang HL, et al. (2012) Anaplastic lymphoma kinase (ALK) regulates initiation of transcription of MYCN in neuroblastoma cells. Oncogene 31: 5193-5200.

10. Berry T, Luther W, Bhatnagar N, Jamin Y, Poon E, et al. (2012) The ALK (F1174L) mutation potentiates the oncogenic activity of MYCN in neuroblastoma. Cancer Cell 22: 117-130.

11. Chesler L, Schlieve C, Goldenberg DD, Kenney A, Kim G, et al. (2006) Inhibition of phosphatidylinositol 3-kinase destabilizes Mycn protein and blocks malignant progression in neuroblastoma. Cancer Res 66: 8139-8146.

12. Zhu S, Lee JS, Guo F, Shin J, Perez-Atayde AR, et al. (2012) Activated ALK collaborates with MYCN in neuroblastoma pathogenesis. Cancer Cell 21: 362373.

13. Heukamp LC, Thor T, Schramm A, De Preter K, Kumps C, et al. (2012) Targeted expression of mutated ALK induces neuroblastoma in transgenic mice. Sci Transl Med 4: 141ra91.

14. Zhang L, Wu B, Baruchel S (2017) Oral metronomic topotecan sensitizes crizotinib antitumor activity in ALKF1174L drug-resistant neuroblastoma preclinical models. Transl Oncol 10: 604-611.

15. Morris SW, Kirstein MN, Valentine MB, Dittmer KG, Shapiro DN, et al. (1994) Fusion of a kinase gene, ALK, to a nucleolar protein gene, NPM, in nonHodgkin's lymphoma. Science 263: 1281-1284.

16. Soda M, Choi YL, Enomoto M, Takada S, Yamashita Y, et al. (2007) Identification of the transforming EML4-ALK fusion gene in non-small-cell lung cancer. Nature 448: 561-566.

17. Hallberg B, Palmer RH (2013) Mechanistic insight into ALK receptor tyrosine kinase in human cancer biology. Nat Rev Cancer 13: 685-700.

18. Mosse YP, Laudenslager M, Longo L, Cole KA, Wood A, et al. (2008) Identification of ALK as a major familial neuroblastoma predisposition gene. Nature 455: 930-935.

19. Chen Y, Takita J, Choi YL, Kato M, Ohira M, et al. (2008) Oncogenic mutations of ALK kinase in neuroblastoma. Nature 455: 971-974.

20. George RE, Sanda T, Hanna M, Fröhling S, Luther W, et al. (2008) Activating mutations in ALK provide a therapeutic target in neuroblastoma. Nature 455 975-978.

21. Bresler SC, Weiser DA, Huwe PJ, Park JH, Krytska K, et al. (2014). ALK mutations confer differential oncogenic activation and sensitivity to ALK inhibition therapy in neuroblastoma. Cancer cell 26: 682-694.

22. Cazes A, Louis-Brennetot C, Mazot P, Dingli F, Lombard B, et al. (2013) Characterization of rearrangements involving the ALK gene reveals a nove truncated form associated with tumor aggressiveness in neuroblastoma. Cancer Res 73: 195-204.

23. Okubo J, Takita J, Chen Y, Oki K, Nishimura R, et al. (2012) Aberrant activation of ALK kinase by a novel truncated form ALK protein in neuroblastoma Oncogene 31: 4667-4676.

24. Maris JM, Weiss MJ, Mosse Y, Hii G, Guo C, et al. (2002) Evidence for a hereditary neuroblastoma predisposition locus at chromosome 16p12-13. Cancer Res 62: 6651-6658

25. Pugh TJ, Morozova O, Attiyeh EF, Asgharzadeh S, Wei JS, et al. (2013) The genetic landscape of high-risk neuroblastoma. Nat Genet 45: 279-284.

26. De Brouwer S, De Preter K, Kumps C, Zabrocki P, Porcu M, et al. (2010). Metaanalysis of neuroblastomas reveals a skewed ALK mutation spectrum in tumors with MYCN amplification. Clin Cancer Res 16: 4353-4362. 
27. Souttou B, Carvalho NB, Raulais D, Vigny M. (2001). Activation of anaplastic lymphoma kinase receptor tyrosine kinase induces neuronal differentiation through the mitogen-activated protein kinase pathway. J Biol Chem 276: 95269531.

28. Zamo A, Chiarle R, Piva R, Howes J, Fan Y, et al. (2002) Anaplastic lymphoma kinase (ALK) activates Stat3 and protects hematopoietic cells from cell death. Oncogene 21: 1038-1047.

29. Kwak EL, Bang YJ, Camidge DR, Shaw AT, Solomon B, et al. (2010) Anaplastic lymphoma kinase inhibition in non-small-cell lung cancer. N Engl J Med 363: 1693-1703.

30. Rodig SJ, Shapiro GI (2010) Crizotinib, a small-molecule dual inhibitor of the c-Met and ALK receptor tyrosine kinases. Curr Opin Investig Drugs 11: 14771490.

31. Cui JJ1, Tran-Dubé M, Shen H, Nambu M, Kung PP, et al. (2011) Structure based drug design of crizotinib (PF-02341066), a potent and selective dual inhibitor of mesenchymal-epithelial transition factor (c-MET) kinase and anaplastic lymphoma kinase (ALK). J Med Chem 54: 6342-6363.

32. Azarova AM, Gautam G, George RE (2011) Emerging importance of ALK in neuroblastoma. Semin Cancer Biol 21: 267-275.

33. Mossé YP, Lim MS, Voss SD, Wilner K, Ruffner K, et al. (2013) Safety and activity of crizotinib for paediatric patients with refractory solid tumours or anaplastic large-cell lymphoma: A Children's Oncology Group phase 1 consortium study. Lancet Oncol 14: 472-480.

34. Bresler SC, Wood AC, Haglund EA, Courtright J, Belcastro LT, et al. (2011) Differential inhibitor sensitivity of anaplastic lymphoma kinase variants found in neuroblastoma. Sci Transl Med 3: 108ra114.

35. Mosse YP, Deyell RJ, Berthold F, Nagakawara A, Ambros PF, et al. (2014) Neuroblastoma in older children, adolescents and young adults: A report from the International Neuroblastoma Risk Group project. Pediatr Blood Cancer 61 627-635.

36. Katayama R, Shaw AT, Khan TM, Mino-Kenudson M, Solomon BJ, et al. (2012) Mechanisms of acquired crizotinib resistance in ALK-rearranged lung Cancers. Sci Transl Med 4: 120ra17.

37. Tanizaki J, Okamoto I, Okabe T, Sakai K, Tanaka K, et al (2012) Activation of HER family signaling as a mechanism of acquired resistance to ALK inhibitors in EML4-ALK-positive non-small cell lung cancer. Clin Cancer Res 18: 62196226.

38. Kim HR, Kim WS, Choi YJ, Choi CM, Rho JK, et al. (2013). Epithelialmesenchymal transition leads to crizotinib resistance in $\mathrm{H} 2228$ lung cancer cells with EML4-ALK translocation. Mol Oncol 7: 1093-1102.

39. Gainor JF, Dardaei L, Yoda S, Friboulet L, Leshchiner I, et al. (2016) Molecular mechanisms of resistance to first- and second-generation ALK inhibitors in ALK-rearranged lung cancer. Cancer Discov 6: 1118-1133.

40. Sasaki T, Okuda K, Zheng W, Butrynski J, Capelletti M, et al. (2010). The neuroblastoma-associated F1174L ALK mutation causes resistance to an ALK kinase inhibitor in ALK-translocated cancers. Cancer Res 70: 10038-10043.

41. Debruyne DN, Bhatnagar N, Sharma B, Luther W, Moore NF, et al. (2016) ALK inhibitor resistance in ALK(F1174L)-driven neuroblastoma is associated with AXL activation and induction of EMT. Oncogene 35: 3681-3691.

42. De Mariano M, Stigliani S, Moretti S, Parodi F, Croce M, et al. (2017) A genomewide microRNA profiling indicates miR-424-5p and miR-503-5p as regulators of ALK expression in neuroblastoma. Oncotarget.

43. Yan X, Kennedy CR, Tilkens SB, Wiedemeier O, Guan H, et al. (2011) Cooperative cross-talk between neuroblastoma subtypes confers resistance to anaplastic lymphoma kinase inhibition. Genes Cancer 2: 538-549.
44. Tucker ER, Danielson LS, Innocenti P, Chesler L (2015) Tackling crizotinib resistance: The pathway from drug discovery to the pediatric clinic. Cancer Res 75: 2770-2774.

45. Infarinato NR, Park JH, Krytska K, Ryles HT, Sano R, et al. (2016) The ALK ROS1 inhibitor PF-06463922 overcomes primary resistance to crizotinib in ALK-driven neuroblastoma. Cancer Discov 6: 96-107.

46. (2014) Ceritinib gains FDA approval for lung cancer Cancer Discov 4: 753-754

47. Wang Y, Wang L, Guan S, et al. (2016) Novel ALK inhibitor AZD3463 inhibits neuroblastoma growth by overcoming crizotinib resistance and inducing apoptosis. Sci Rep 6: 19423

48. Friboulet L, Li N, Katayama R, et al. (2014) The ALK inhibitor ceritinib overcomes crizotinib resistance in non-small cell lung cancer. Cancer Discov 4: 662-673.

49. Tchekmedyian N, Ali SM, Miller VA, Haura EB (2016). Acquired ALK L1152R Mutation confers resistance to ceritinib and predicts response to alectinib. J Thorac Oncol 11: e87-88.

50. Lu J, Guan S, Zhao Y, Yu Y, Woodfield SE, et al. (2017). The second-generation ALK inhibitor alectinib effectively induces apoptosis in human neuroblastoma cells and inhibits tumor growth in a TH- MYCN transgenic neuroblastoma mouse model. Cancer Lett 400: 61-68.

51. Krytska K, Ryles HT, Sano R, Raman P, Infarinato NR, et al. (2016) Crizotinib synergizes with chemotherapy in preclinical models of neuroblastoma. Clin Cancer Res 22: 948-960.

52. Acker T, Plate KH (2004) Hypoxia and hypoxia inducible factors (HIF) as important regulators of tumor physiology. Cancer Treat Res 117: 219-248.

53. Semenza GL (2000) HIF-1: mediator of physiological and pathophysiological responses to hypoxia. J Appl Physiol (1985) 88: 1474-1480.

54. Martinengo C, Poggio T, Menotti M, Scalzo MS, Mastini C, et al. (2014) ALK dependent control of hypoxia-inducible factors mediates tumor growth and metastasis. Cancer Res 74: 6094-6106.

55. Timur M, Akbas SH, Ozben T (2005) The effect of Topotecan on oxidative stress in MCF-7 human breast cancer cell line. Acta Biochim Pol 52: 897-902.

56. Beppu K, Nakamura K, Linehan WM, Rapisarda A, Thiele CJ (2005) Topotecan blocks hypoxia-inducible factor-1alpha and vascular endothelial growth factor expression induced by insulin-like growth factor-I in neuroblastoma cells. Cancer Res 65: 4775-4781.

57. Rapisarda A, Zalek J, Hollingshead M, Braunschweig T, Uranchimeg B, et al. (2004) Schedule- dependent inhibition of hypoxia-inducible factor-1alpha protein accumulation, angiogenesis and tumor growth by topotecan in U251HRE glioblastoma xenografts. Cancer Res 64: 6845-6848.

58. Wood AC, Krytska K, Ryles HT, Infarinato NR, Sano R, et al. (2017) Dual ALK and CDK4/6 inhibition demonstrates synergy against neuroblastoma. Clin Cancer Res 23: 2856-2868.

59. Wang HQ, Halilovic E, Li X, Liang J, Cao Y, et al. (2017) Combined ALK and MDM2 inhibition increases antitumor activity and overcomes resistance in human ALK mutant neuroblastoma cell lines and xenograft models. Elife 6.

60. Lodrini M, Sprüssel , Astrahantseff K, Tiburtius D, Konschak R, et al. (2017) Using droplet digital PCR to analyze MYCN and ALK copy number in plasma from patients with neuroblastoma. Oncotarget.

61. Tucker ER, Tall JR, Danielson LS, Gowan S, et al. (2017) Immunoassays fo the quantification of ALK and phosphorylated ALK support the evaluation of ontarget ALK inhibitors in neuroblastoma. Mol Oncol 11: 996-1006.

62. Padovan-Merhar OM, Raman P, Ostrovnaya I, Kalletla K, Rubnitz KR, et al. (2016) Enrichment of targetable mutations in the relapsed neuroblastoma genome. PLoS Genet 12: e1006501. 